\title{
De nouveaux profils pour des professionnels manquants?
}

Académie Suisse des Sciences Médicales ASSM

\begin{abstract}
Les personnalités suivantes ont fait partie du groupe de travail qui avait rédigé le rapport en 2007 pour le compte du groupe de pilotage «La médecine en Suisse demain»: Dr Werner Bauer, Küsnacht (président) Prof. Anne-Françoise Allaz, Genève; Prof. Charles Bader Genève; Barbara Gassmann, Berne; lic. oec. Pius Gyger, Zurich; Dr Jacques de Haller Berne; Dr Romy Mahrer Imhof, Bâle; Dr Peter Tschudi, Bâle; Priska Wildhaber Isler, Küsnacht.

\section{En plus des membres du} groupe de travail, les personnalités suivantes ont participé à l'élaboration du commentaire en 2011: Dr Hermann Amstad, ASSM, Bâle; lic. phil. Nadja Birbaumer, ASSH, Berne; Prof. Claudine Burton-Jeangros, Genève; Dr Magdalena Rosende, Lausanne; Prof. Ursula Streckeisen, Berne; Prof. Peter Suter, ASSM, Presinge.

Le rapport de l'année 2007 et le commentaire 2011 sont également publiés comme brochure; celle-ci peut être obtenue gratuitement auprès de l'ASSM (mail@samw.ch).
\end{abstract}

Correspondance:

Académie Suisse des Sciences

Médicales ASSM

Petersplatz 13

CH-4051 Bâle

mail@samw.ch
Fin 2007, l'Académie Suisse des Sciences Médicales (ASSM) publia le rapport «Les futurs profils professionnels des médecins et des infirmiers dans la pratique ambulatoire et clinique» dans le Bulletin des médecins suisses (46/2007, p. 1942-52) [1]; aucune brochure qui aurait pu être distribuée ne fut imprimée à l'époque, contrairement aux autres rapports publiés dans le cadre du projet «La médecine en Suisse demain».

Le 17 juin 2009, l'ASSM organisa à Berne la journée de réflexion consacrée aux «Mutations des professions de soins: points de vue externes et internes» dans l'esprit d'une certaine durabilité; par ailleurs, des représentants de l'Académie suisse des sciences humaines et sociales (ASSH) firent partie du comité du programme.

Lors du congrès, les orateurs et les participants étaient d'accord pour reconnaître que seule une coopération étroite entre les nombreuses professions de la santé - au sein des équipes interprofessionnelles allait permettre une assistance médicale optimale et que l'acceptation mutuelle ainsi que le respect des compétences spécifiques représentaient une condition essentielle.

Presque quatre ans après la publication du rapport «Les futurs profils professionnels des médecins et des infirmiers dans la pratique ambulatoire et clinique», le groupe de travail d'alors, augmenté des experts de l'ASSH faisant partie du comité du programme, s'est retrouvé pour présenter une rétrospective et procéder à un examen des perspectives et possibilités de contribuer à l'application des recommandations émises à l'époque. Une des mesures décidées concerne la publication inchangée du rapport de 2007 (quasiment à la manière d'un «document historique»), auquel on ajoute un commentaire actuel (c'est-à-dire le présent commentaire) [2, 3] .

\section{Le système de santé change lentement, mais continuellement}

Le système suisse de santé n'est pas resté le même depuis 2007. On peut citer ici l'introduction des DRG (ou forfaits par cas), l'application du financement des soins et l'élaboration d'une loi sur la prévention. Par ailleurs, il faut mentionner la création de l'association «Médecins de famille Suisse», le dépôt de l'initiative sur la médecine de famille ainsi que l'accueil favorable du projet Managed Care au Parlement.

Plusieurs évolutions déjà nommées dans le rapport se sont encore accentuées depuis, notamment la féminisation de la profession de médecin et les besoins croissants en personnel dans le domaine de la santé. L'étude de l'OBSAN publiée en 2009 ainsi que le rapport de la CDS et de l'OdASanté sur les besoins en effectifs, concernant les futurs besoins en personnel soignant, ont également secoué la politique et conduit à de nombreuses interventions. Les avis étaient unanimes sur le fait qu'il n'est pas judicieux de combler les pénuries de personnel par l'arrivée de professionnels étrangers et de contribuer ainsi à la migration internationale dans le secteur de la santé.

\section{La mutation des professions sous I'angle des sciences sociales}

Le rapport se focalise pour l'essentiel sur deux groupes professionnels: les médecins et les infirmiers dans la pratique ambulatoire et clinique. Toutes les autres professions du secteur thérapeutique et surtout celles qui se rapportent à la promotion de la santé sont mentionnées de manière plutôt accessoire, en quelque sorte comme partenaires des deux professions concernées ici. Pour obtenir une perspective plus détaillée et «plus complète» des professions de la santé, il y a lieu de réfléchir à la nécessité d'envisager un prochain rapport qui mettrait l'accent sur d'autres «professions médicales», comme par exemple celles dont les clients ne sont pas des malades (ou «patients»), mais des personnes en bonne santé. Les nombreuses activités liées à la prévention des risques étendent sans cesse le domaine du système de santé et conduisent à ce que la totalité de la population (personnes en bonne santé et malades) se retrouve face à une multiplicité croissante de professionnels de la santé.

Si le rapport se concentre largement sur les professionnels, il s'agit cependant de ne pas perdre de vue les patients, situés au centre des activités du système de santé. Il faudrait également mentionner que la complexité croissante du système de soins rend difficile la compréhension d'un système hermétique, en particulier aux patients atteints d'une maladie chronique; il en résulte une dépendance accrue des patients par rapport aux médecins et aux infirmiers ou aux interruptions de traitement et de prise en charge.

Les attentes et comportements des patientes et des patients sont influencés par les appartenances sociales (couche sociale; tranche d'âge; sexe) et les systèmes de valeurs. Cette diversité ainsi que l'impor- 
tance des facteurs sociaux doivent être considérées au moment de décrire la prise en charge adéquate d'un patient.

\section{L'économicisation de la médecine comme moteur?}

Le fait de leur donner une orientation déterminée par la gestion a graduellement transformé de nombreux hôpitaux, foyers, etc. en entreprises. Les institutions sociales sont dirigées selon des principes à l'origine en vigueur pour des usines. Au-delà des effets positifs liés à cette évolution (conception économique des déroulements, etc.), il faut également examiner les conséquences qui ne manquent pas de soulever des questions et de déclencher des controverses.

Quel sens donner, pour la profession de médecin, au fait que des médecins en milieu hospitalier deviennent des «employés ordinaires»? Ou que le contrôle de la qualité, les entretiens personnels, etc. deviennent une évidence dans les hôpitaux? Quelle signification accorder aux actions syndicales menées par des médecins? Si le «caractère de la profession» a tendance à régresser, la perte de prestige n'est pas la seule concernée; il est également question ici de ce qui se produit avec la relative et traditionnelle autonomie professionnelle du médecin. Il faut par ailleurs se demander quelle importance attribuer aux associations professionnelles, face aux conditions qui ont changé: au-delà de la poursuite «d'intérêts corporatifs», elles sont toujours occupées à formuler des principes éthiques qui doivent, en définitive, garantir un exercice de la médecine et des soins de bonne qualité.

Quelle signification accorder au fait que les patients deviennent des «clients»? Qu'en est-il lorsqu'ils sont appelés à devenir des patients «responsables» (empowerment)? Il semble y avoir des images sociales d'un patient qui contrôle activement lui-même - à la manière d'un entrepreneur - sa visite chez le médecin et son quotidien, en accord avec les principes d'un «mode de vie sain». De telles représentations sontelles liées à des évolutions émancipatrices ou au contraire à une mise au pas croissante que les médecins et les infirmiers peuvent/doivent soutenir? Lorsque le rapport constate que «l'élément de base reste la relation personnelle et souvent de longue durée qui existe entre le médecin et son patient», il importe de se demander si cet élément de base est soutenu ou menacé par les développements récents.

Une économicisation fulgurante du côté des professionnels est plus visible qu'il y a encore quatre ans, en partant des contribuants et de la politique: elle s'accompagne d'excès (administration, repérage et recensement des données/indices, mesures de contrôle) pour aboutir à une qualité des résultats qu'on peut de moins en moins qualifier d'adéquate. La situation est problématique lorsque la qualité du résultat est interprétée de manière exclusivement financière et au sens de la réduction des coûts, et que des qualités difficilement mesurables telles que «les soins fiables pour quelqu'un» sont négligées.
Il convient de se demander si la défense acharnée des rôles et modèles traditionnels dont il est question dans le rapport, ne présente qu'un caractère exclusivement problématique. Le scepticisme des médecins - à l'égard de la gestion de leur entreprise, par exemple - n'est pas nécessairement et uniquement motivé par une soif de pouvoir; au contraire, il est concevable que l'on aspire à une relation adéquate au patient et que celle-ci se trouve perturbée par des intérêts propres à l'entreprise.

\section{Conflits et conséquences: où en sommes-nous aujourd'hui?}

La question se pose de savoir si des évolutions au caractère presque exceptionnel «se produisent» simplement au sens d'une tendance toujours plus forte ou si ces développements peuvent être introduits, encouragés, accélérés ou dirigés - et par qui? Qui doit devenir actif: Les associations professionnelles? L'Etat? Des scientifiques qui prêtent une oreille attentive ou encore des pionniers ou des organisations de pionniers isolés? Comment concilier les intérêts et les revendications, en partie légitimes, des groupes concernés avec les impératifs liés à la transformation? Jusqu'où doit-on accepter que les changements entraînent toujours des gagnants et des perdants et comment garantir que les patientes et les patients ne seront pas les perdants? Où le marché a-t-il sa place et qu'est-ce qui continue à faire partie d'un «service public»?

L'élucidation de ces questions serait d'autant plus urgente que la situation de pénurie chez les médecins de famille et le personnel infirmier ne s'est d'aucune façon désamorcée et que tous les pronostics indiquent une aggravation supplémentaire.

Même si les possibilités et chances d'une nouvelle répartition des responsabilités au sein des professions de la santé continuent d'être nommées et discutées, peu de choses concrètes se sont produites ces dernières années pour soutenir une transformation des modèles et des profils professionnels. Si un large consensus semble exister, au moins dans les opinions, sur la nécessité pour les contenus, conditions cadres et compétences des professions de la santé, de se transformer et de se développer, il ne dépasse pas le stade d'une relative incertitude.

Des commentaires courts concernant l'état de l'application ou de la non-application de certaines conséquences concrètes, discutées de manière détaillée dans le rapport, s'imposent en 2011:

\section{Il est nécessaire de redéfinir les compétences et responsabilités des médecins et soignants, au sein des groupes professionnels}

Une telle redéfinition des domaines professionnels doit avoir pour objectif de veiller à ce que chaque groupe professionnel puisse apporter sa contribution spécifique au meilleur système de soins possible, dans le cadre de ses compétences de base. Cela se produit d'une part au sein des institutions prises séparément 


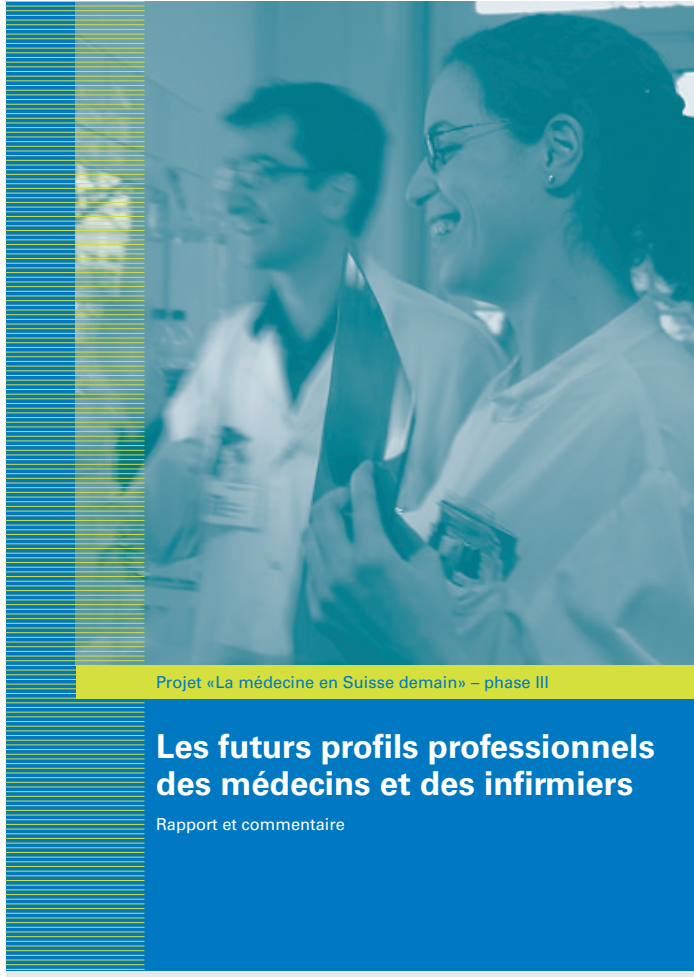

Cet article est un commentaire du rapport de l'année 2007.

et dans le cadre de réglementations libérales qui garantissent avant tout la qualité. Des incitations, des conditions cadres optimisées (par ex. au niveau de la législation) et des mesures de soutien sont toutefois nécessaires pour permettre la réalisation de nouveaux modèles. Les médecins et les infirmiers ainsi que les autres groupes professionnels doivent apprendre à accepter les déplacements de leurs compétences. En échange, ils peuvent attendre une réduction des charges bureaucratiques, une concentration sur l'essentiel de leur activité ainsi qu'une meilleure qualité de travail. Des projets isolés sont arrivés jusqu'au grand public depuis la publication du rapport, sans que l'on puisse constater une évolution marquée dans le sens d'une nouvelle interprofessionnalité.

\section{La formation au niveau baccalauréat universitaire} procurera, à l'avenir, les mêmes éléments communs de base, aussi bien aux médecins qu'aux soignants

Nous constatons que la plupart des facultés de médecine ainsi que de nombreuses institutions de formation pour le personnel infirmier continuent d'être occupées à plein temps par l'organisation de leurs programmes respectifs. La réalisation du modèle de Bologne exige notamment les plus grands efforts de la part des facultés. Il est néanmoins nécessaire de se raccrocher au but consistant à procurer aux futurs médecins et infirmiers au minimum certains blocs de formation communs qui leur transmettent une langue commune, des principes ainsi que la base de connaissances portant sur les caractéristiques de la santé et de la maladie.
De nouveaux modèles d'activité transdisciplinaire en équipe, dans laquelle les professions individuelles peuvent être installées en fonction de leurs compétences de base, doivent être encouragés

L'introduction à grande échelle de tels modèles (cabinets de groupe interprofessionnels, centres de santé, «maisons de santé centrées sur le patient») exige plus qu'un soutien verbal et la bienveillance de politiciens et de journalistes. Ces dernières années, des efforts concrets n'ont pas pu être observés, bien qu'il apparaisse de plus en plus clairement que les chances de trouver une relève suffisante dépendent exclusivement d'une correspondance réussie entre la vie professionnelle et les représentations d'un choix de vie satisfaisant. Même si le nombre des cabinets de groupe augmente, il ne s'agit bien souvent pas de modèles véritablement intégrés, mais de collectifs de médecins partageant la même adresse et le souci parfaitement légitime de faire baisser leurs frais, le plus souvent sans autre forme d'échange pour améliorer les soins aux patients. Il est réjouissant de constater qu'il existe malgré tout des groupes de pionniers qui vont encore plus loin et que certaines communes commencent également à soutenir des projets de ce type, faute de quoi l'assistance médicale de base ne serait plus du tout assurée dans leur secteur. Si de telles possibilités de «partenariat entre le public et le privé» sont encore trop rares, elles constituent néanmoins des signes positifs vers de nouvelles évolutions. En revanche, la reprise de cabinets et de centres médicaux par des assureurs-maladie ne représente pas un développement souhaitable, car elle s'oppose à la «séparation des pouvoirs» dans le secteur de la santé et mène nécessairement à des conflits d'intérêts insolubles.

\section{Les associations professionnelles sont chargées d'introduire les mesures nécessaires et d'assurer un dialogue constructif}

Mis à part des contacts amicaux mais superficiels, il n'est pas (encore) possible de constater une collaboration ciblée. Si le système de milice - qui conduit à une surcharge maximale des associations professionnelles et sociétés spécialisées, même sans de tels projets peut contribuer à faire reculer devant une problématique neuve et difficile à traiter, celle-ci est toutefois inévitable!

\section{Il est nécessaire de disposer d'un nombre suffisant de places accréditées de formation postgraduée dans les hôpitaux ou les cabinets et d'assez d'emplois à temps partiel}

Les formations prégraduée et postgraduée représentent des investissements pour le futur et pas uniquement des coûts susceptibles d'être réduits. Nous n'aurons, à vrai dire, plus à nous inquiéter des profils professionnels, des modèles tournés vers l'avenir et d'une qualité élevée des soins, si nous ne parvenons pas à garantir la formation prégraduée et postgraduée, au 
niveau de la quantité et de la qualité. Il ne serait plus alors question que de plans d'urgence, au mieux!

\section{Le baccalauréat universitaire doit devenir - y compris en Suisse alémanique et au Tessin - le diplôme normal du personnel soignant}

Plusieurs raisons sous-tendent cette revendication: les exigences posées au personnel soignant continuent de croître, tandis que la structure des professions de santé est soumise à de profonds changements. De nouvelles professions ont été introduites - «Assistante/Assistant en santé et soins communautaires» et «Infirmière praticienne» - qui sont exercées sous la surveillance et la direction de personnel infirmier diplômé.

Des investissements adéquats en matière de formation sont essentiels pour l'assistance médicale de notre population.

\section{Il faut intensifier le recrutement de la relève}

Quoi qu'il arrive dans ce domaine, les progrès sont encore insuffisants; en effet, il ne suffit pas d'intensifier le recrutement, car il ne peut naturellement pas produire les résultats escomptés sans un nombre suffisant de places d'études. Si l'intention est d'étendre et d'académiser la formation du personnel soignant de manière à lui permettre d'assumer un domaine de compétences plus large, il faut considérer qu'il ne sert pas à grand-chose de remplacer une profession en manque de personnel (médecin) par une autre (infirmier). Des mesures complémentaires sont nécessaires, au même titre que davantage de personnes intéressées par les professions de la santé qui seront alors en mesure de s'épanouir à différents niveaux.

En conséquence, les dispositions légales et les tarifs doivent être révisés de manière à éviter qu'ils ne constituent un obstacle aux modifications des compétences et des responsabilités Les développements attendus se poursuivront plus difficilement ou seront même empêchés par l'absence de conditions cadres favorables qui devraient par ailleurs inclure une certaine fonction incitative. Les adaptations tarifaires sont indispensables, de même que la possibilité pour les infirmiers ou d'autres groupes professionnels d'exercer une activité en tant que prestataires indépendants. La loi devrait permettre à ces intervenants de fournir des prestations couvertes par l'assurance de base, sous leur propre responsabilité et dans un cadre clairement défini.

\section{Perspectives: I'avenir de la médecine est à la fois tourné vers l'équipe, interprofessionnel et modulaire}

Le rapport publié en 2007 a ouvert le débat portant sur des sujets importants, relatifs aux identités professionnelles et à leurs transformations. Il décrit un ensemble de possibilités et de mesures permettant de continuer à développer positivement un secteur de la santé toujours encore en bon état de fonctionnement. Le personnel de la santé, à la fois compétent et engagé, joue un rôle déterminant dans ce contexte. Les réflexions économiques ne doivent pas venir pervertir ou refouler les demandes très anciennes de l'humanité ayant trait aux problématiques éthiques. L'espoir de trouver de bonnes solutions est permis aussi longtemps que des personnes défendront autrui et la bonne cause.

Cela fait presque dix ans que l'ASSM attire régulièrement l'attention sur la pénurie sensible et croissante de médecins et de personnel soignant dans notre pays. Si ce fut tout d'abord un phénomène auquel on prêta peu attention ou que l'on considéra comme un «problème de répartition» entre la ville et la campagne, il en va tout autrement aujourd'hui. Tout à coup, tout le monde est du même avis: les hôpitaux universitaires ressentent la pénurie, la FMH donne l'alarme et les médecins de famille montent sur les barricades. Tous réclament des mesures énergiques, et en particulier davantage de places de formation pour les médecins et les autres professionnels de la santé dans les facultés et les hautes écoles spécialisées.

En Suisse, entre un tiers et la moitié de l'ensemble des professionnels du secteur médical viennent aujourd'hui de l'étranger. Entre-temps, les organisations professionnelles, les politiciens et les médias sont également alarmés en Allemagne, le «principal pays fournisseur» du personnel manquant en Suisse. La pénurie y est aussi perceptible et le nombre des postes inoccupés en milieu hospitalier augmente rapidement. L'Allemagne ne pourra pas éviter les mesures efficaces pour freiner l'émigration du personnel médical - et ensuite?

La large reconnaissance du problème menaçant constitue à vrai dire une bonne nouvelle - mieux vaut tard que jamais. Mais comme pour d'autres diagnostics tardifs, cela n'est pas fait pour simplifier la thérapie. Des remèdes plus puissants s'imposent maintenant, la correction du déficit exigera davantage de temps, les complications ne sont pas exclues et les chances de guérison ne sont pas les meilleures. La seule augmentation du nombre des places de formation ne nous permettra pas de remédier à la situation désastreuse. Des mesures courageuses supplémentaires sont nécessaires, en particulier pour colmater le «pipeline qui fuit», c'est-à-dire limiter autant que possible la perte de professionnels qualifiés. Il s'agit de tenir davantage compte des valeurs et des idées des jeunes générations, pour permettre notamment aux femmes de concilier à long terme les objectifs de vie et les buts professionnels. Les femmes constituant d'ores et déjà la majorité des professionnels de la santé, chaque réforme doit tenir compte de cette réalité.

La tentative visant à écarter le dommage du secteur de la santé aurait vraisemblablement échoué, si un commentaire du rapport sur les profils professionnels devait aboutir en 2015 à de telles conclusions! 